\title{
LES PLANTES UTILISÉES PAR LES ABEILLES AU TCHAD MÉRIDIONAL
}

\author{
Die Bienenpflanzen des südlichen Tschad
}

\author{
Claude GADBIN \\ Laboratoire de Botanique historique et Palynologie, \\ Faculté des Sciences et Techniques Saint-Jérôme, \\ rue Henri-Poincaré, 13397 Marseille Cedex 4
}

\begin{abstract}
RÉSUMÉ
Cette étude réalise un début d'inventaire des plantes utilisées par Apis mellifica adansonii, au Tchad méridional. Trois méthodes d'approche : Enquêtes éthnologiques, observations èthologiques, analyses polliniques, sont utilisées pour appréhender ce domaine de connaissances à peu près inexploré actuellement.

Quarante-cinq taxons mellifères sont répertoriés, avec un niveau de précision atteignant l'espèce dans $50 \%$ des cas. Quelques taxons remarquables sont signalés : les Graminées; les nymphéacies et $P_{\text {sidium }}$ gayava. Les taxons déterminés par l'analyse pollinique reflètent la composition floristique régionale, en particulier la structure des spectres polliniques des miels étudiés semble corrélative de la variété floristique du lieu de récolte; cependant, le choix de l'abeille au sein de la flore régionale demeure un élément prépondérant. La fluctuation saisonnière des compositions polliniques des miels reflète la phénologie régionale et particulièrement son absence de synchronisation au sein même d'une espèce.

Cette étude contribue à approcher certaines données de l'éthologie, l'écologie et la biologie d'Apis mellifica adansoni en zone sahélo-soudanienne, au Tchad. Les informations rassemblées seraient utilisables dans une perspective de modernisation de l'apiculture locale.
\end{abstract}

\section{SUMM ARY}

\section{PLANTS USED BY THE HONEYBEES IN SOUTHERN TCHAD}

In the present paper an attempt is made to inventory the melliferous plants used by Apis mellifica adansonii in southern Tchad. It was made possible thanks to ethnological enquiries, ethological observations and pollen analyses. 45 melliferous taxa could be identified and their species defined for $50 \%$ of them. 
Some remarkable taxa are recorded: Graminaeae, Nympheaceae, Psidium gayara. The taxa determined with pollen analysis reflect the local floristic composition; in particular the structure of pollen spectra in the studied honeys seem to be related to the floristic variability in the collecting place; but the choice made by the honey-bee in the local flora is a preponderant factor. Seasonal variations in the pollinic composition of honeys are linked with the local phenology and especially with its lack of synchronisation within the same species.

This study is an approach to some ethological, ecological and biological data concerning Apis mellifica adansonii in the Sahelo-soudanian region of Tchad. The information presented here could be of interest in a modernization project of Tchadian bee-keeping.

\section{INTRODUCTION}

En Afrique, l'abeille domestique (Apis mellifica L.) est connue et utilisée depuis des temps reculés, comme en témoignent les peintures préhistoriques rupestres retrouvées à Ebusingata en Afrique du Sud et figurant la récolte du miel par les Boschimans (GuY, 1972). Par contre, l'étude scientifique de sa biologie, son éthologie, son écologie, ainsi que des pratiques apicoles n'a été réalisée que très partiellement sur l'immense continent africain. Les connaissances acquises sur Apis mellifica en Afrique sont caractérisées par leur disparité de niveau et leur discontinuité géographique.

Le seul ouvrage de synthèse sur l'apiculture tropicale est celui de SMITH (1960) qui donne une liste de 10 familles ou genres mellifères présents au Tchad méridional. Dubois et Collard décrivent en 1950, au Congo, une flore mellifère dont 9 genres sont représentés dans la flore du Tchad. Le rapport de LePISSIER (1968) sur l'apiculture en République Centrafricaine établit une liste de 25 espèces mellifères de la forêt tropicale, présentes aux frontières sud du Tchad.

Plus récemment, la thèse de N'DiAYE (1974) apporte quelques précisions sur différents taxons de plantes mellifères sénégalaises. Parmi celles-ci, 16 espèces spontanées au Sénégal existent dans la région tchadienne que nous avons étudiée. Le travail de SowUNmI (1976) donne une information précise sur les variations de la représentation pollinique dans les miels du sud Nigéria. Parmi les espèces récoltées dans cette zone de végétation beaucoup plus méridionale que notre lieu de travail, on n'en trouve que quatre qui soient très communes. Enfin, la synthèse publiée par CRANE (1973) sur les plantes mellifères donne la liste la plus complète actuellement de ces espèces africaines. Les quelques informations existantes sur les rapports d'Apis mellifica avec des végétations apparentées à celle des pays qui nous occupent sont éparses et sous la forme d'études spécifiques du rôle pollinisateur de l'abeille vis-à-vis de plantes cultivées, dont certaines seulement sont présentes au Tchad (CHANDLER et MDEMU, 1974; CHAPMAN, 1964; Free, 1976, Mc Gregor, 1974; Beyleveld, 1968-a).

Parfois, une seule espèce botanique est considérée, et le rôle d'Apis mellifica dans sa pollinisation est défini : Carica papaya L. (Allan, 1963; FreE, 1974), Ficus et Ziziphus spina Christi I. Willd. (Galil \& Zeroni, 1967), Arachis hypogaea L. (HAMMONS \& LeUCK, 1966), Gossypium (Mc Gregor, 1974; MaHadevan \& Chandy, 
1959). Enfin, de rares travaux sur l'apiculture mentionnent la collecte du pollen par l'abeille sur quelques plantes : au Sénégal (LINDER, 1967; N'DiAYE, 1974), en Angola (Rosario Nuñes \& Tordo, 1960), au Transvaal (Beyleveld, 1968 b); de mêne dans les travaux de Crane (1973), SMITH (1957) et SowUnmi (1976).

\section{LE MILIEU}

\section{A. - Le cadre géographique}

La présente étude est limitée à une région de $50 \mathrm{~km}$ autour de N'Djamena. Incluse dans un triangle d'une superficie un peu supérieure à $1000 \mathrm{~km}^{2}$, dont le côté nord-est va du Chari au Barh Linia, elle s'étire en pointe entre les lits du Logone et du Chari au sud; son côté ouest suit grossièrement la frontière camerounaise (Fig. 1).
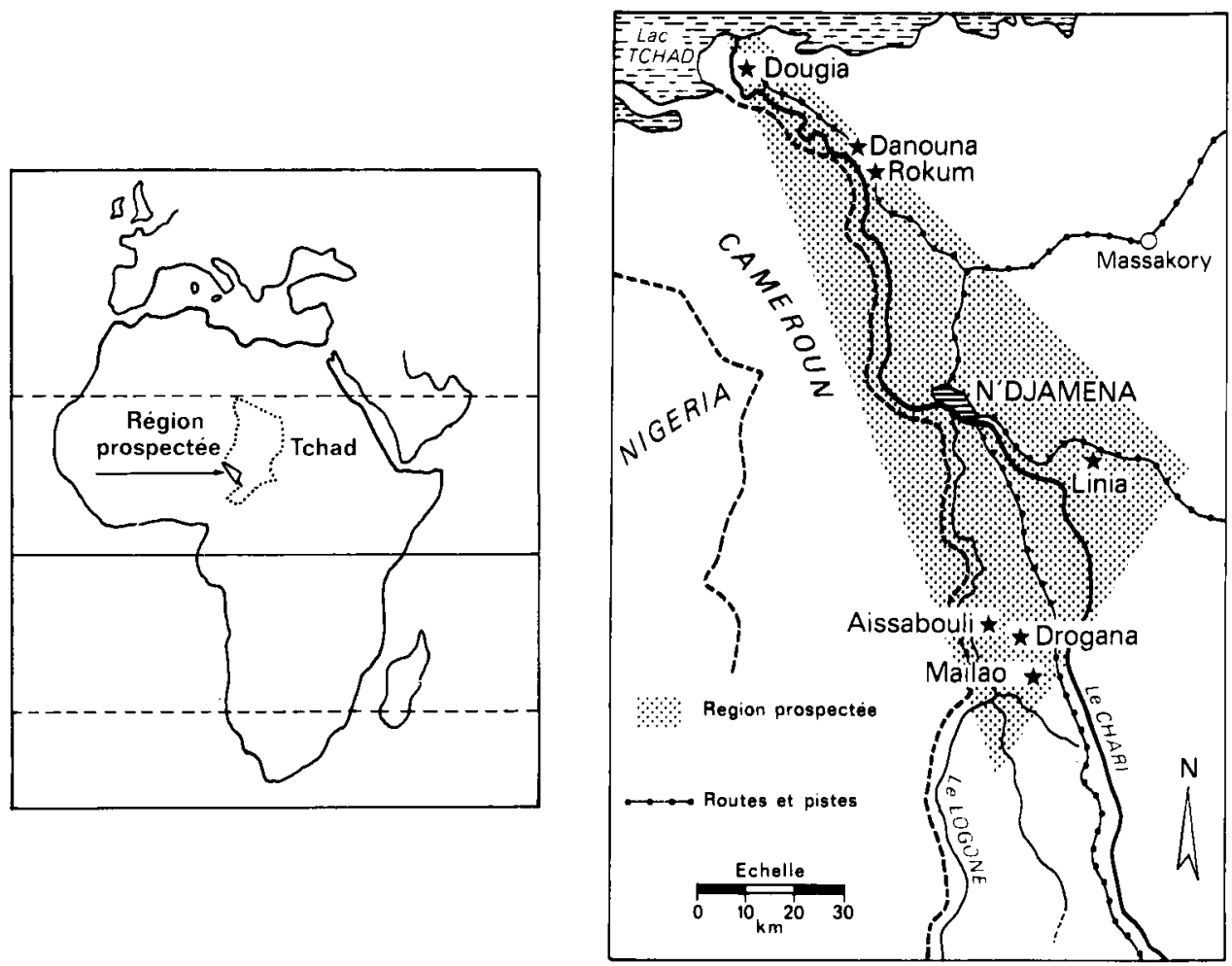

FIG. 1 a et b. - Cartes de situation.

Aв8. 1. - Karte des Gebietes. 
Cette région, qui s'étend entre les latitudes nord $12^{\circ} 30$ et $11^{\circ} 40$, est située dans la zone sahélo-soudanienne caractérisée au point de vue climatique (AUBREVILLE, 1949) par une pluviosité annuelle comprise entre 950 et $500 \mathrm{~mm}$ à répartition estivale. Elle porte un couvert végétal de type savane arbustive boisée qui, localement, est caractérisée par sa structure en mosaïque où voisinent forêts claires à Combretacées dominantes, forêts claires en bouquets à Khaya senegalensis, savanes boisées et arbustives d'origine anthropique, steppes ou fourrés à Mimosées, rôneraies le long des fleuves, et, enfin, forêts "galeries" le long du Chari (Fotıus, 1973).

La région étudièe est très marquée par la présence de cours d'eau permanents : le Chari et le Logone, qui vont se déverser au nord dans le lac Tchad. Lors des crues, leurs eaux inondent largement les rives; celles-ci demeurent submergées pendant plusieurs mois. Le Logone prend naissance sur le plateau de l'Adamaoua, au Cameroun, à $1200 \mathrm{~m}$; dans son cours inférieur, au confluent avec le Chari, il traverse des formations quaternaires récentes. Il déborde abondamment sur les deux rives et recouvre d'eau les plaines d'inondations nommées "yaérés ".

\section{B. - La végétation}

La couverture végétale, assez pénétrable, est formée de petits arbustes ou d'arbres, occupant des sols hydromorphes, des sols ferrugineux tropicaux peu lessivés, des sols bruns ou bruns-rouges subarides. Les espèces les plus fréquemment observées sont : Acacia senegal, A. scorpioides, Piliostigma sp., Balanites aegyptiaca, Boscia senegalensis, Guiera senegalensis, Ziziphus mauritania, Cadaba farinosa, Acacia seyal.

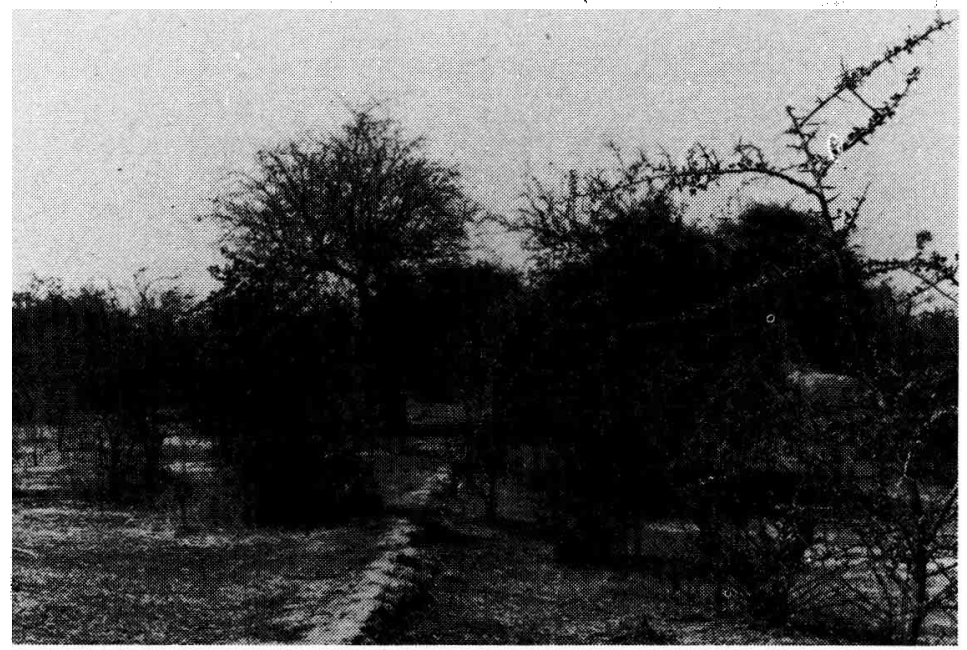

Fig. 2 a. - Couverture végétale. Groupement à Acacias, modifié par l'influence humaine.

(présence de Balanites aegyptiaca, Guiera senegalensis, visibles ici).

Авв. 2 а. - Vegetation. Gruppen von Akazien, verändert durch menschlichen Einfluss

(z. B. die auf dem Bilde sichtbaren

Balanites aegyptiaca, Guiera senegalensis). 
Cette végétation présente des variations locales liées à la nature du sol, à l'hydrographie, à la proximité des cours d'eau ou de la nappe phréatique.

Le groupement à Acacia seyal et Echinochloa colonum sa retrouve partout. De type steppique, il se développe sur sols argileux. C'est le plus fréquent dans cette zone. Il est favorisé par l'action anthropozoïque qui tasse le sol et permet à Acacia seyal, se reproduisant par graines, d'assurer sa dissémination, malgré l'émondage des branches maîtresses pratiqué, en saison sèche, par les bergers qui rendent ainsi accessibles au bétail, les gousses mûres.

Associée à cette formation, au sud, une savane boisée à Anogeissus leïocarpus et Andropogon gayanus se développe sur sol sableux profond. Elle est caractérisée par la présence de Combretacées nombreuses où Anogeissus domine. La strate ligneusé est très souvent modifiée par l'action humaine : $A$. leiocarpus disparait au profit de Combretum hypopilinum, Guiera senegalensis, Detarium microcarpum, Balanites aegyptia$c a$; alors que la strate herbacée tend vers une composition stable où dominent : Hyparrhenia baguirmica, Loudeita togoensis, Eragrostis tremula, Mitracarpus scaber.

Dans la partie nord de la zone d'étude on rencontre une formation steppique de sol argileux dite à Acacia nilotica et Echinochloa sp. (GASTON, 1976), caractérisée pour les ligneux par Acacia nilotica et Mitragyna inermis, pour les herbacées par Echinochloa stagmina, Ipomoea aquatica, Orysa sp., Limnophyton obtusifolium.

Sur la limite est du domaine d'étude, outre la steppe à Acacia seyal, existe une steppe herbeuse à Sesbania et Sorghum, qui apparait sur les bas fonds inondables à sol d'argile noire.

Toutes ces formations possèdent des bons représentants situés sur les termitières. Celles-ci présentent, en fonction de la nature du sol, de la latitude, du degré d'hydromorphie, une grande variété. On y retrouve toujours certaines espèces qui s'y développent préférentiellement, parmi lesquelles les ligneux : Tamarindus indica, Balanites aegyptiaca, Diospyros mespiliformis, Combretum glutinosum, etc.

Enfin, cette région, fortement peuplée, est très marquée par l'action humaine: "L'homme exerce une influence profonde sur la composition floristique, condamnant certaines espèces, en favorisant d'autres, éventuellement introduisant de nouvelles plantes " (LETOUZEy, 1968, p. 57). L'influence anthropique, partout évidente, diffère suivant les ethnies qui l'exercent. Certaines, dont les représentants sont cultivateurs, éleveurs, pêcheurs et chasseurs cultivant le mil rouge, s'installent en ordre dispersé et se déplacent assez fréquemment. Ils entraînent une dégradation de la végétation naturelle sur de grandes surfaces mais laissent derrière eux se reconstituer des savanes arbustives secondaires où ne persistent que Faidherbia albida, Hyphaene thebaica, Ficus gnaphalocarpa...

D'autres ethnies, au contraire, se groupent en gros villages fixes, cultivant divers mils, Arachis hypogaea, Gossypium et Sesamum indicum en auréoles concentriques s'éloignant de plus en plus du village au fur et à mesure de l'augmentation de la popula- 


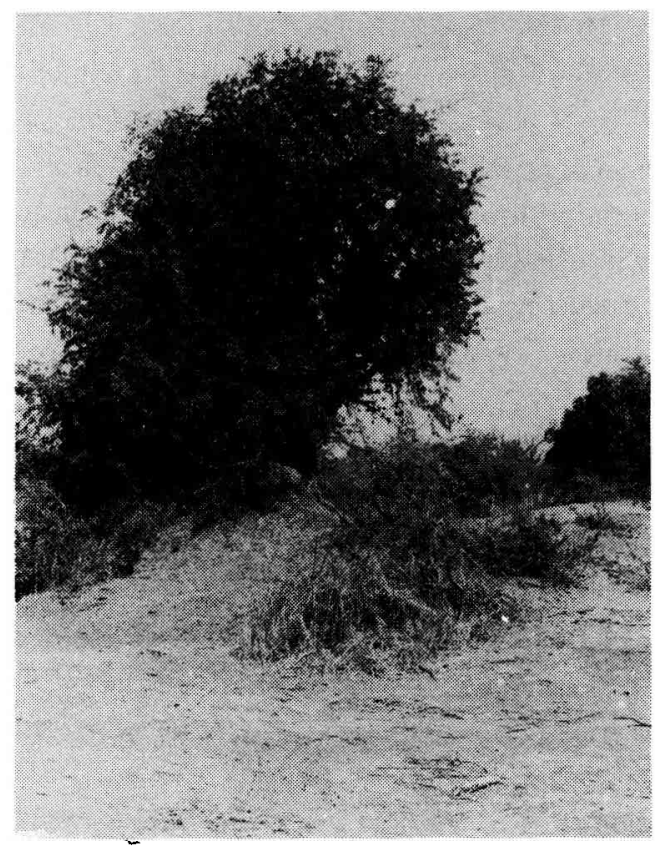

F1G. 2 b. - Couverture végétale. Sur termitière. Ici Tamarindus indica.

Aвв. 2 b. - Vegetation. Auf dem Termitenhügel Tamarindus indica.

tion et de l'épuisement des terres. La modification du couvert végétal est beaucoup plus profonde car il y a destruction des espèces vivaces. Le faciès de jachère persiste longtemps, le phénomène est aggravé par l'habitude de ces ethnies de s'installer le long des axes de communication où les terres de culture confluent, provoquant la destruction presque totale des grands arbres de part et d'autre des axes routiers ou des fleuves.

Au milieu de ses cultures l'homme isole Acacia albida, Balanites aegyptiaca, Celtis integrifolia, Ficus platyphilla, Khaya senegalensis, Kigelia africana, Tamarindus indica, Ziziphus spina-christi alors qu'à l'extérieur des champs, sur les terres de pâture ou dans les jachères, les éléments sahéliens, épineux surtout, font leur apparition (LETOUZEY, 1968).

\section{MATÉRIEL ET MÉTHODES}

La détermination des plantes mellifères est essentiellement le résultat des analyses polliniques auxquelles ont été soumis les échantillons de miels récoltés et les réserves de pollen extraites de rayons fournis par les apiculteurs. Elle résulte cependant aussi d'observations directes sur les abeilles butineuses et d'informations provenant des apiculteurs régionaux. 


\section{1) Choix des échantillons de miel}

Les miels provenaient, soit de récoltes faites avec l'aide d'apiculteurs aux trois villages de Linia, Maïlo et Drogana, soit d'achats effectués chaque semaine pendant une année sur le marché de Ndjamena, où quelques vendeuses de miel sont présentes en permanence. La provenance probable des miels achetés fut toujours demandée aux vendeuses. Bien que ce miel commercialisé soit celui pour lequel nous possédons le plus grand nombre d'échantillons, les résultats de leur analyse pollinique n'ont pas été utilisés car un simple inventaire qualitatif préalable des espèces présentes a montré des taxons originaires de zones très inégalement éloignées de Ndjamena, correspondant à des milieux très différents comme les montagnes camerounaises, par exemple. L'utilisation de tels miels aurait nécessité la connaissance précise des réseaux de distribution de la denrée vendue. Une première approche dans la région de Dougia a révélé leur complexité : l'importance de la récolte, l'occasion d'un déplacement de l'apiculteur vers la capitale ou vers le Cameroun frontalier sont susceptibles d'intervenir de façon totalement imprévisible.

Les résultats présentés seront donc limités à l'analyse des 17 échantillons de miels dont la récolte a été effectuée au sein d'un rucher localisé avec rigueur, dans une zone de végétation. connue (cf. annexe).

\section{2) Préparation des échantillons de miel}

Les miels analysés ont été obtenus par pressage manuel des rayons. Ce type d'extraction, traditionne] au Tchad, a été également employé lors des récoltes personnelles, dans un souci d'homogénéité des méthodes d'échantillonnage. Cette technique introduit une distorsion quantitative très importante dans la représentation pollinique au sein des miels. En effet, lors du pressage, les pollens stockés en alvéoles se mêlent aux pollens présents dans le miel en proportions indéterminables.

\section{3) Réserves de pollen}

Les réserves polliniques receuillies lors des cueillettes de miel, et simplement extraites à la main des alvéoles de cire les contenant, sont dispersées par écrasement, traitées selon les mêmes méthodes que les miels, et leur contenu pollinique analysé.

\section{4) Pollens de référence}

$\mathrm{Au}$ sein de la flore tchadienne environ 1500 espèces sont actuellement recensées (LEBRUN et al., 1972). L'identification des pollens présents dans les miels étudiés suppose donc la possession d'une collection suffisante de pollens de référence. Le choix des espèces retenues a été fait en utilisant les informations bibliographiques et les relevés botaniques déjà établis.

Les espèces végétales présentes sur les sites apicoles et supposées mellifères ont permis de constituer le premier fichier « Plantes supposées mellifères " dans lequel les taxons ont été regroupés par familles botaniques. Sur chaque fiche ont été notées :

- les informations résultant de l'établissement d'une fiche phénologique de terrain,

- les informations d'intérêt apicole : présence de nectaires, observations de butinage par des insectes, référence bibliographique comportant citation de la plante comme mellifère.

Pour chaque pollen retenu une fiche morphologique a été établie. Elle comporte une description aussi précise que possible du pollen appuyée par des photographies au microscope photonique.

La collecte du pollen a été faite autant que possible sur des plantes fraîches, sur le terrain.

En l'absence d'échantillon "frais ", les pollens ont été prélevés sur les herbiers locaux.

Cent soixante fiches de ce type ont pu être réalisées.

\section{5) Préparation des pollens de référence et des miels}

Le choix de la technique de traitement et de montage des pollens de référence et des miels a posé quelques problèmes.

Dans un premier temps les pollens de référence ainsi que les miels ont été traités selon les méthodes classiques en mélissopalynologie (Maurizio et Louveaux, 1965), mais lors des analyses, il fut quasiment 
impossible d'identifier de façon rigoureuse les pollens contenus dans les miels. C'est pourquoi nous avons eu recours à l'acétolyse selon ERDTMAN (1969) avec une méthode que nous avons mise au point (GADBIN, 1979) et qui figure dans la dernière version des méthodes de la mélisso-palynologie (Louveaux et al., 1978).

6) Numération des grains de pollen des miels

VERGERON (1964) a montré qu'il est nécessaire de compter à peu près 1200 grains dans un miel pour avoir une représentation pollinique satisfaisante. Pour les miels tchadiens, dont la composition pollinique est a priori totalement inconnue, un test sur l'apparition de nouvelles espèces et sur leur représentativité en fonction du nombre de grains comptés a été effectué. Le miel choisi pour cet essai a été récolté en mai 1975 au village de Rokum. On n'observe plus d'apparition de nouveaux taxons au-delà du comptage de 500 grains (Fig. 3, Tabl. 1). Les proportions relatives des grains ne se modifient que très faiblement au-delà de ce chiffre.

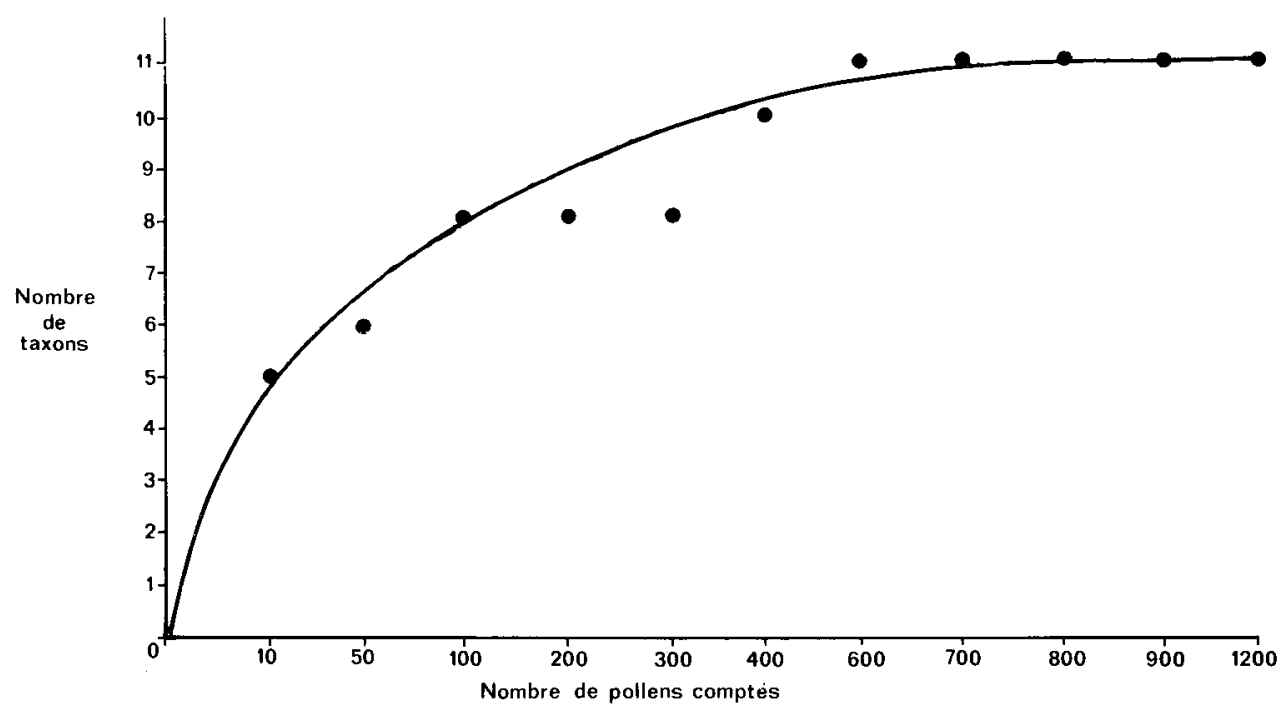

FIG. 3. - Variation du nombre de taxons déterminés en fonction du nombre de pollens comptés.

Aв8. 3. - Variation der Zahl der determinierten Taxa

in Bezug auf die Anzahl augezählter Pollenkörner.

7) Niveau de détermination des pollens

L'identification des pollens a été poursuivie aussi loin que possible. Cependant, pour diverses raisons, le niveau atteint est variable : famille, genre ou espèce.

Les pollens dominants, ainsi que les pollens d'accompagnement ont toujours été identifiés au niveau spécifique; cette détermination a posé parfois quelques problèmes, surtout pour les espèces présentant une grande variabilité morphologique intraspécifique. Ce fut le cas pour l'Anacardiacée Lannea velutina. Cette espèce, si elle possède toujours la même sculpture striée de l'exine offre une grande variabilité dans l'épaisseur, la densité, et la sinuosité des stries. Ce phénomène a été nettement observé dans le miel de Linia (récolte de mai 1976). Cette variabilité est un fait fréquemment observable chez les espèces tropicales (MALEY : comm. orale) (Fig. 4). 


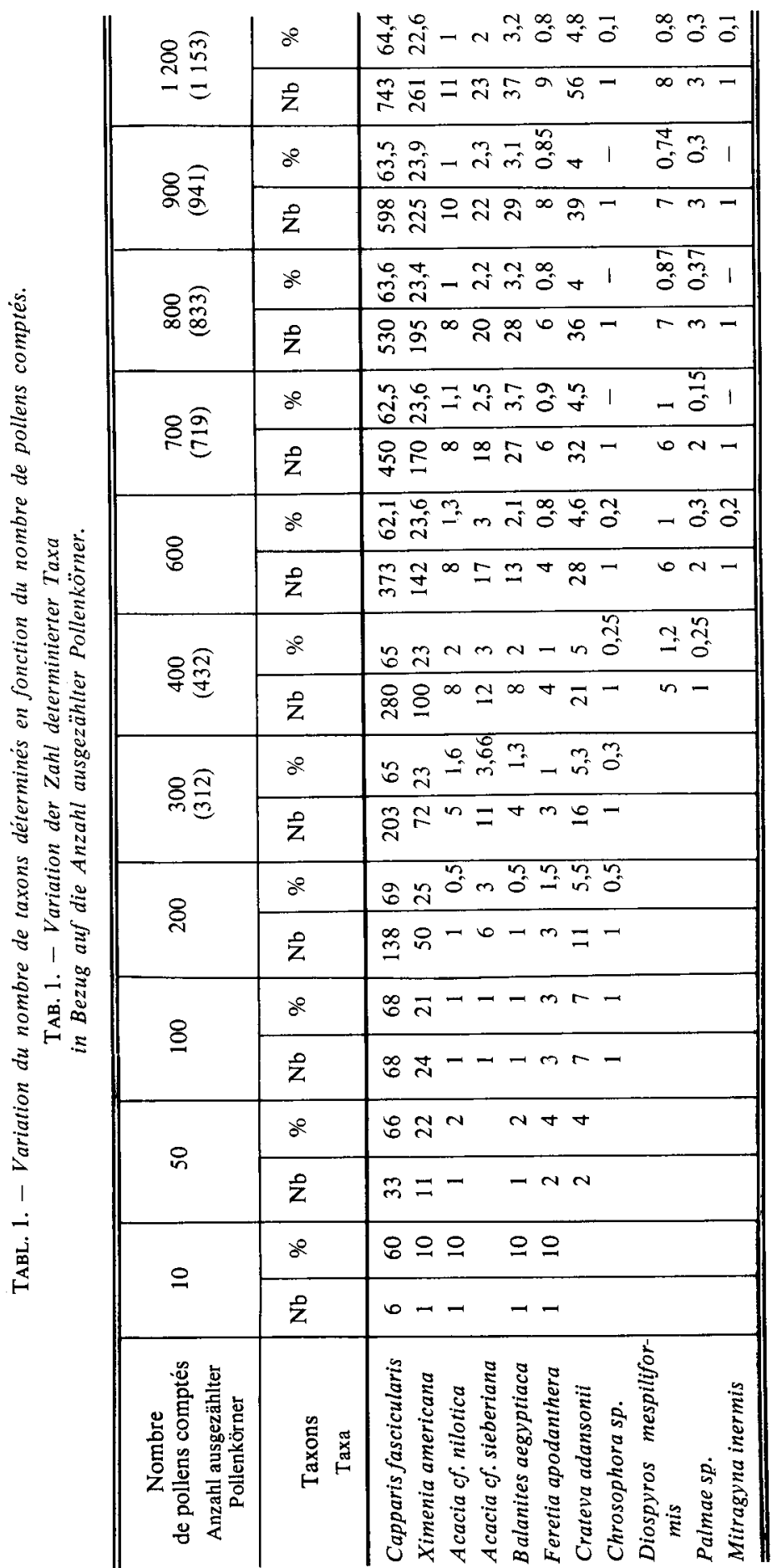



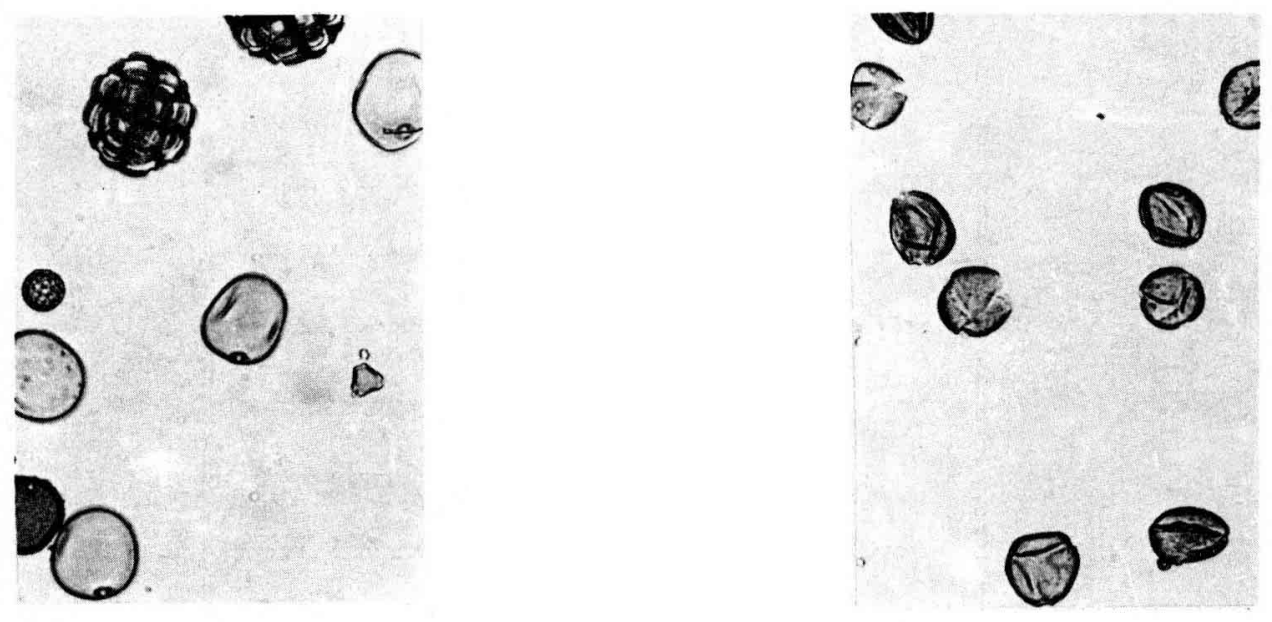

FIG. 4. - Miels acétolysés $(\times 300)$.

A gauche : miel de Linia à graminées dominantes. A droite: miel de Linia à Lannea velutina.

Aвв. 4. - Azetolysierte Honige ( $\times 300)$.

Links : Honig aus Linia mit Vorherrschen von Graminaceen.

Rechts : Honig von Linia mit Lannea velutina.
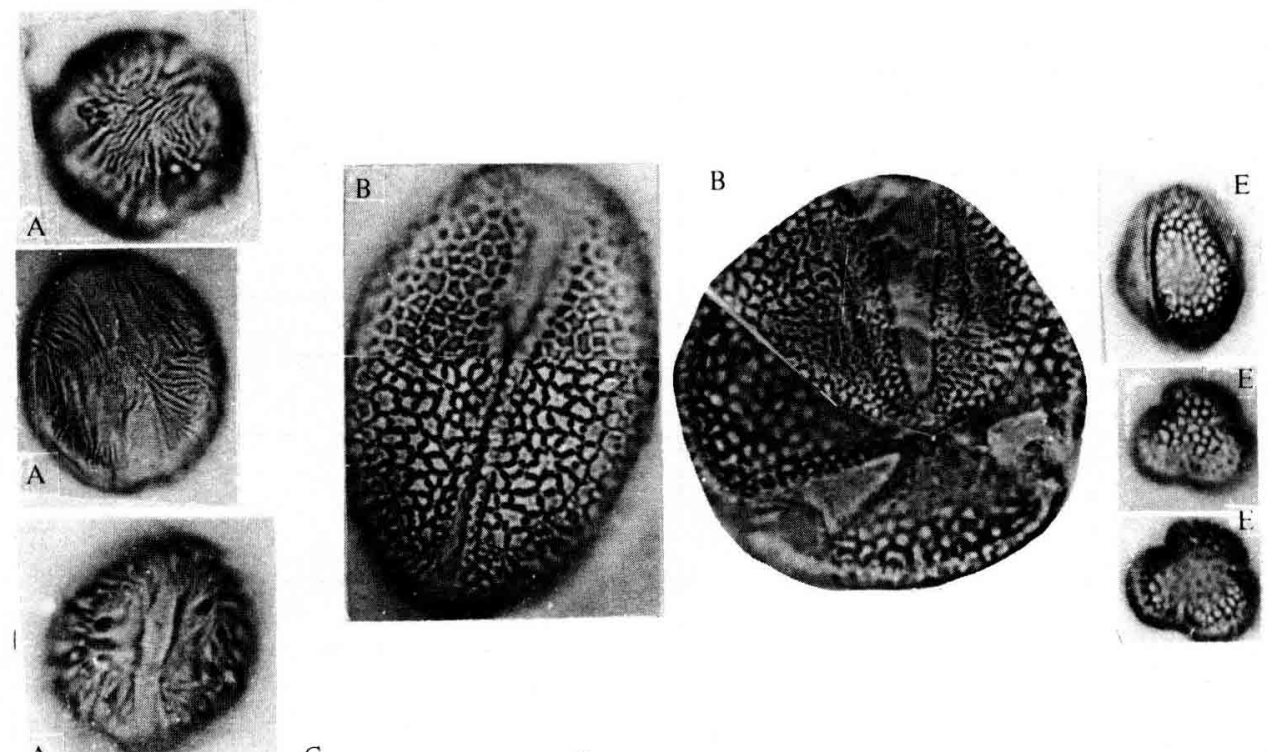

A
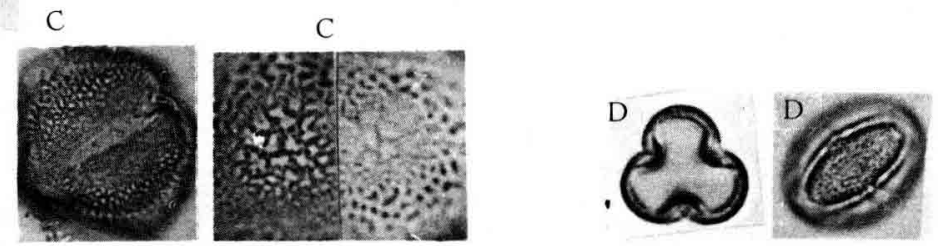

Fig. 5. - Pollens de plantes mellifères $(\times 1000)$.

A) Anacardiaceae : Lannea velutina,

B) Bignoniaceae : Kigelia africana,

C) Caesalpiniaceae : Bauhinia rufescens,

D) Capparidaceae : Capparis fascicularis,

E) Salicaceae : Salix cotuleoides.

AвB. 5. - Pollen von Bienenpflanzen. 
Lorsque dans le genre identifié une seule espèce est signalée par les botanistes, dans la zone étudiée, elle est indiquée à titre de référence dans la mesure où sa morphologie pollinique ne s̀y oppose pas. Il faut remarquer que, dans ce cas, les abeilles ont très bien pu visiter un taxon proche, rare et non signalé dans la flore locale. C'est le cas de l'Ebénacée : Diospyros mespiliformis, seule espèce connue au Tchad, alors que 4 espèces ont été inventoriées au Sénégal. Enfin, dans le cas de genres comportant plusieurs espèces signalées au sud du Tchad, mais dont l'une est représentée en quantité importante sur le terrain étudié, le nom de celle-ci est retenu, dans la mesure où le pollen de référence de cette espèce l'autorise, ainsi en est-il pour la Rubiacée : Mytragyna inermis.

Certains pollens n'ont été identifiés qu'au niveau générique, la collection de référence ne renfermant pas toutes les espèces signalées dans la zone avoisinant le rucher, ce qui rend l'identification rigoureuse impossible. C'est le cas, par exemple, des Ziziphus (Rhamnacées) désignées dans les comptages seulement par le nom générique.

Cette identification rigoureuse au niveau du genre, est suivie parfois d'une indication spécifique exprimée sous la forme "cf. ". Ainsi les Nympheaceae de type $N y$ mphea sont parfois désignées sous le terme Nymphea cf. lotus, lorsque le pollen de Nympheaceae identifié s'apparente à l'espèce $N$. lotus seule possédée en collection, le genre $N y m p h e a$ étant représenté par trois espèces dans la région étudiée.

Pour les Graminées et les Palmées la détermination s'arrête à la famille. Les Graminées rencontrées dans les miels étudiés appartenaient au type "Graminées spontanées ", voisines des Andropogonées (BONNEFILLE, 1972). Pour la famille des Palmées, qui n'est représentée que par deux espèces au sud du Tchad, le comptage est rapporté au nom de l'espèce, seule observée aux abords du rucher étudié : à Linia, le palmier doum (Hyphaena thebaïca), à Mailao le palmier rhônier (Borassus aethiopum).

\section{8) Expression des résultats}

Une fiche signalétique des 17 miels retenus porte les indications suivantes : lieux, date et conditions de la récolte, et caractères physico-chimiques du miel. Y figurent également les résultats qualitatifs et quantitatifs de l'analyse pollinique, sous la forme d'un spectre pollinique établi selon les critères retenus par les mélisso-palynologues d'Europe.

Les taxons identifiés étant regroupés en : " pollens dominants ” (figurant à plus de $45 \%$ dans la préparation); " pollens d'accompagnement " (présents de $15 \%$ à $45 \%$ dans la préparation); " pollens isolés " (moins de $15 \%$ des pollens comptés). Ces derniers sont répartis en deux sous-groupes :

- présents de + de $1 \%$;

- présents de - de $1 \%$.

Ces chiffres ont été établis après un comptage de 1000 à 1200 pollens, chiffre qui exclut toute distorsion quantitative due au nombre de grains comptés.

Ce comptage est réalisé sur un balayage systématique de toute la préparation.

On trouve en annexe 1 l'ensemble des 17 fiches correspondant aux analyses effectuées.

\section{RÉSULTATS}

\section{1) Approche ethnologique}

Le questionnaire d'enquête "Apiculture au Tchad " (GADBIN, 1976) comporte un paragraphe " Plantes mellifères ". Les données fournies par ce questionnaire concernent huit espèces connues traditionnellement comme mellifères par les ethnies locales. Elles sont regroupées dans le tableau 2. 
TABL. 2. - Plantes mellifères connues traditionnellement.

TAB. 2. - Traditionell bekannte Honigpflanzen.

\begin{tabular}{|c|c|c|c|}
\hline $\begin{array}{l}\text { Plantes mellifères } \\
\text { Honigpllanzen }\end{array}$ & $\begin{array}{l}\text { Arabes } \\
\text { Araber } \\
\text { Yessie } \\
\text { (Linia) }\end{array}$ & $\begin{array}{l}\text { Arabes } \\
\text { Arabes } \\
\text { Amadjie } \\
\text { Babalia } \\
\text { (Donouna) } \\
\text { (Rokoun) }\end{array}$ & $\begin{array}{l}\text { Kotoko } \\
\text { (Drogona) }\end{array}$ \\
\hline $\begin{array}{l}\text { ASCLEPIADACEAE } \\
\text { Calotropis procera }\end{array}$ & & + & \\
\hline $\begin{array}{l}\text { CAESALPINIACEAE } \\
\text { Tamarindus indica }\end{array}$ & + & + & \\
\hline $\begin{array}{l}\text { EBENACEAE } \\
\text { Diospyros mespiliformis }\end{array}$ & + & + & \\
\hline $\begin{array}{l}\text { MIMOSACEAE } \\
\text { Acacia sp. }\end{array}$ & + & + & + \\
\hline $\begin{array}{l}\text { RHAMNACEAE } \\
\text { Ziziphus mauritania }\end{array}$ & & + & \\
\hline $\begin{array}{l}\text { RUBIACEAE } \\
\text { Mitragyna inermis }\end{array}$ & + & + & + \\
\hline $\begin{array}{l}\text { ULMACEAE } \\
\text { Celtis integrifolia }\end{array}$ & + & & \\
\hline $\begin{array}{c}\text { ZYGOPHYLLACEAE } \\
\text { Balanites aegyptiaca }\end{array}$ & + & + & + \\
\hline
\end{tabular}

2) Observations éthologiques

Le butinage d'abeilles domestiques a été observé sur les espèces suivantes (Tabl. 3).

3) Les plantes sources de pollen

Les réserves de pollen étudiées renferment 20 taxons différents appartenant à 14 familles, parmi lesquelles celles des Capparidacées et des Rubiacées sont remarquables par le grand nombre d'espèces trouvées. Parmi celles-ci toutes les 'espèces présentes en quantité importante le sont sur deux sites au plus, aucune n'ayant été trouvée sur les trois sites. Ces divergences peuvent résulter de manière aléatoire de la non simultanéité des récoltes et de leur nombre limité.

Cependant, ces analyses permettent d'établir une liste des espèces végétales butinées pour la récolte du pollen par Apis mellifica adansonii au sud du Tchad (Tabl. 4).

Cette liste est remarquable par sa briéveté : 20 taxons seulement. Elle a été établie sur trop peu d'échantillons pour être exhaustive et ne couvre pas un cycle de végétation complet. 
TABL. 3. - Liste des espèces sur lesquelles les abeilles butinent.

TAв. 3. - Liste der von Bienen beflogenen Pflanzen.

\begin{tabular}{|c|c|c|}
\hline $\begin{array}{l}\text { Famille } \\
\text { Familie }\end{array}$ & $\begin{array}{l}\text { Genre } \\
\text { Gattung }\end{array}$ & $\begin{array}{c}\text { Espèces } \\
\text { Art }\end{array}$ \\
\hline $\begin{array}{l}\text { ANACARDIACEAE } \\
\text { BIGNONIACEAE }\end{array}$ & $\begin{array}{l}\text { Anacardium } \\
\text { Kigelia } \\
\text { Tecoma }\end{array}$ & $\begin{array}{l}\text { occidentale } \\
\text { africana } \\
\text { stans }\end{array}$ \\
\hline CAESALPINIACEAE & $\begin{array}{l}\text { Bauhinia } \\
\text { Caesalpinia } \\
\text { Detarium } \\
\text { Piliostigma } \\
\text { Tamarindus }\end{array}$ & $\begin{array}{l}\text { rufescens } \\
\text { pulcherina } \\
\text { microcarpum } \\
\text { reticulatum } \\
\text { indica }\end{array}$ \\
\hline CAPPARIDACEAE & $\begin{array}{l}\text { Cadaba } \\
\text { Crateva }\end{array}$ & $\begin{array}{l}\text { farinosa } \\
\text { adansonii }\end{array}$ \\
\hline COCHLOSPERMACEAE & Cochlospermum & tinctorium \\
\hline CONVOLVULACEAE & Ipomoea & aquatica \\
\hline EUPHORBIACEAE & $\begin{array}{l}\text { Euphorbia } \\
\text { Ricinus }\end{array}$ & $\begin{array}{l}\text { pulcherina } \\
\text { communis }\end{array}$ \\
\hline LILIACEAE & Chlorophytum & tuberosum \\
\hline MALVACEAE & $\begin{array}{l}\text { Hibiscus } \\
\text { Gossypium }\end{array}$ & $\begin{array}{l}\text { diversifolius } \\
\text { hirsutum }\end{array}$ \\
\hline MELIACEAE & Azadirachta & indica \\
\hline MIMOSACEAE & Acacia & \\
\hline OLACACEAE & Ximenia & americana \\
\hline PAPILIONACEAE & Dalbergia & sisoo \\
\hline RHAMNACEAE & Ziziphus & mauritania \\
\hline RUBIACEAE & $\begin{array}{l}\text { Mitragyna } \\
\text { Gardenia }\end{array}$ & inermis \\
\hline RUTACEAE & Citrus & sinensis \\
\hline
\end{tabular}

\section{4) Les plantes mellifères}

Le pollen trouvé dans le miel stocké témoigne de l'activité de récolte du nectar des fleurs par les abeilles : lorsqu'une plante est visitée par une abeille, l'absorption du nectar s'accompagne de celle des pollens contenus dans celui-ci. En effet, très souvent le pollen des anthères, lorsqu'il est dispersé, tombe sur les nectaires de la plante (MAURIঙ zıo, 1968). Par contre, le pollen mis en réserve directement est récolté en tant que tel par l'insecte indépendamment du nectar. Dans toutes les analyses il sera très difficile de distinguer, parmi les plantes fréquentées par les abeilles, celles qui sont une source de nectar de celles qui approvisionnent la ruche en pollen ou sur lesquelles les abeilles effectuent les deux types de récoltes. Les pollens identifiés permettront donc seulement de conclure à la nature mellifère de l'espèce végétale en cause. Seuls quelques cas particuliers permettront de préciser ses véritables rapports avec l'abeille.

La compilation de l'ensemble des analyses polliniques des miels permet d'établir une liste de plantes en relation avec Apis mellifica au Tchad. Cette liste de 28 familles 
TABL. 4. - Liste des espèces pollinifères.

TAB. 4. - Liste der Pollenpflanzen.

\begin{tabular}{|c|c|}
\hline $\begin{array}{l}\text { Famille } \\
\text { Familie }\end{array}$ & $\begin{array}{l}\text { Genre et espèce } \\
\text { Gattung und Art }\end{array}$ \\
\hline ACANTHACEAE & Hygrophila \\
\hline ANACARDIACEAE & Lannea cf. velutina \\
\hline BIGNONIACEAE & Kigelia africana \\
\hline CAPPARIDACEAE & $\begin{array}{l}\text { Capparis corymbosa } \\
\text { Capparis fascicularis } \\
\text { Cadaba farinosa } \\
\text { Crateva adansonii }\end{array}$ \\
\hline CAESALPINIACEAE & $\begin{array}{l}\text { Bauhinia rufescens } \\
\text { Piliostigma cf. reticulatum }\end{array}$ \\
\hline COMBRETACEAE & type Combretum \\
\hline COMPOSEAE & type Centaurea \\
\hline EBENACEAE & Diospyros mespiliformis \\
\hline GRAMINEAE & \\
\hline MELIACEAE & Kaya senegalensis \\
\hline MIMOSACEAE & $\begin{array}{l}\text { Acacia nilotica } \\
\text { Acacia seyal } \\
\text { Acacia sieberiana }\end{array}$ \\
\hline $\begin{array}{l}\text { NYMPHEACEAE } \\
\text { PALMAE }\end{array}$ & Nymphaea cf. lotus \\
\hline RUBIACEAE & $\begin{array}{l}\text { Mitragyna inermis } \\
\text { Feretia apodanthera } \\
\text { Morelia senegalensis }\end{array}$ \\
\hline
\end{tabular}

(Tabl. 5) regroupe 45 taxons. Alors que le nombre de miels analysés (17) est très supéricur au nombre de réserves de pollen étudiées (6), le nombre de taxons représenté est encore remarquablement faible en regard du nombre d'espèces végétales présentes dans cette région. Cette donnée confirme l'intervention d'un choix très sélectif de l'abeille parmi les espèces végétales utilisables alentour de la ruche.

De l'observation de ce tableau il ressort la dominance qualitative de la famille des Capparidaceae, qui est représentée par 6 espèces différentes, celles des Rubiaceae, des Euphorbiaceae et des Mimosaceae, dont 4 espèces sont présentes.

L'observation des spectres polliniques de l'ensemble des miels fait apparaitre un certain nombre d'espèces mellifères dont les pollens sont fréquents, mais toujours en petit nombre (moins de $15 \%$ ). Ces espèces représentent 18 taxons, dont 16 ont été identifiés dans les stocks de pollens. La famille des Mimosacées, qui est dominante avec celle des Capparidacées, est connue pour être nectarifère (DE VRIEs, 1975). On peut émettre l'hypothèse que ces espèces, fréquentes, mais présentes en petites quantités, sent butinées non seulement pour leur pollen, mais aussi pour leur nectar (Tabl. 6). Cette hypothèse ne serait vérifiable que par l'étude de miels extraits par centrifugation sans pollution par le contenu des cellules à pollen. 
TABL. 5. - Plantes mellifères.

TAB. 5. - Bienenpflanzen.

\begin{tabular}{|c|c|}
\hline $\begin{array}{l}\text { Famille } \\
\text { Familie }\end{array}$ & $\begin{array}{l}\text { Genre et espèce } \\
\text { Gattung und Art }\end{array}$ \\
\hline AMARANTHACEAE & cf. Celosia \\
\hline ANACARDIACEAE & Lannea velutina \\
\hline BIGNONIACEAE & Kigelia africana \\
\hline CAPPARIDACEAE & $\begin{array}{l}\text { Cadaba farinosa } \\
\text { Capparis corymbosa } \\
\text { Capparis fascicularis } \\
\text { Crateva adansonii } \\
\text { Crateva religiosa } \\
\text { Maerua }\end{array}$ \\
\hline CARRYOPHILACEAE & Glinus \\
\hline CAESALPINIACEAE & $\begin{array}{l}\text { Bauhinia rufescens } \\
\text { Piliostigma reticulatum } \\
\text { Piliostigma thoningii }\end{array}$ \\
\hline COMBRETACEAE & $\begin{array}{l}\text { Combretum cf.paniculatum } \\
\text { Combretum }\end{array}$ \\
\hline $\begin{array}{l}\text { CONVOLVULACEAE } \\
\text { COMPOSACEAE }\end{array}$ & Merremia aegyptiaca \\
\hline EBENACEAE. & Diospyros mespiliformis \\
\hline EUPHORBIACEAE & $\begin{array}{l}\text { Chrozophora } \\
\text { Hymenocardia acida } \\
\text { Jatropha } \\
\text { Ricinus communis }\end{array}$ \\
\hline GRAMINACEAE & \\
\hline MALVACEAE & $\begin{array}{l}\text { Abutilon } \\
\text { Hibiscus }\end{array}$ \\
\hline MELIACEAE & $\begin{array}{l}\text { Azadirachta indica } \\
\text { Khaya senegalensis }\end{array}$ \\
\hline MIMOSACEAE & $\begin{array}{l}\text { Acacia nilotica } \\
\text { Acacia seyal } \\
\text { Acacia sieberiana } \\
\text { Mimosa pigra }\end{array}$ \\
\hline MYRTACEAE & Psidium gayal'a \\
\hline NYMPHEACEAE & Nymphea ef lotus \\
\hline OLACACEAE & Xymenia americana \\
\hline PALMAE & $\begin{array}{l}\text { Borassus aethiopum } \\
\text { Hyphaene thebaica }\end{array}$ \\
\hline PAPILIONACEAE & $\begin{array}{l}\text { Dalbergia sisoo } \\
\text { Sesbania }\end{array}$ \\
\hline POLYGONACEAE & Polygonum \\
\hline PORTULACEAE & Portulaca \\
\hline RHAMNACEAE & Ziziphus \\
\hline RUBIACEAE & $\begin{array}{l}\text { Feretia apondanthera } \\
\text { Gardenia } \\
\text { Mitragyna inermis } \\
\text { Morelia senegalensis }\end{array}$ \\
\hline SALIXACEAE & Salix cotuleoïdes \\
\hline SAPINDACEAE & Paullinia pinnata \\
\hline TILLIACEAE & Corchorus fascicularis \\
\hline ZYGOPHYLLACEAE & Balanites aegyptiaca \\
\hline
\end{tabular}




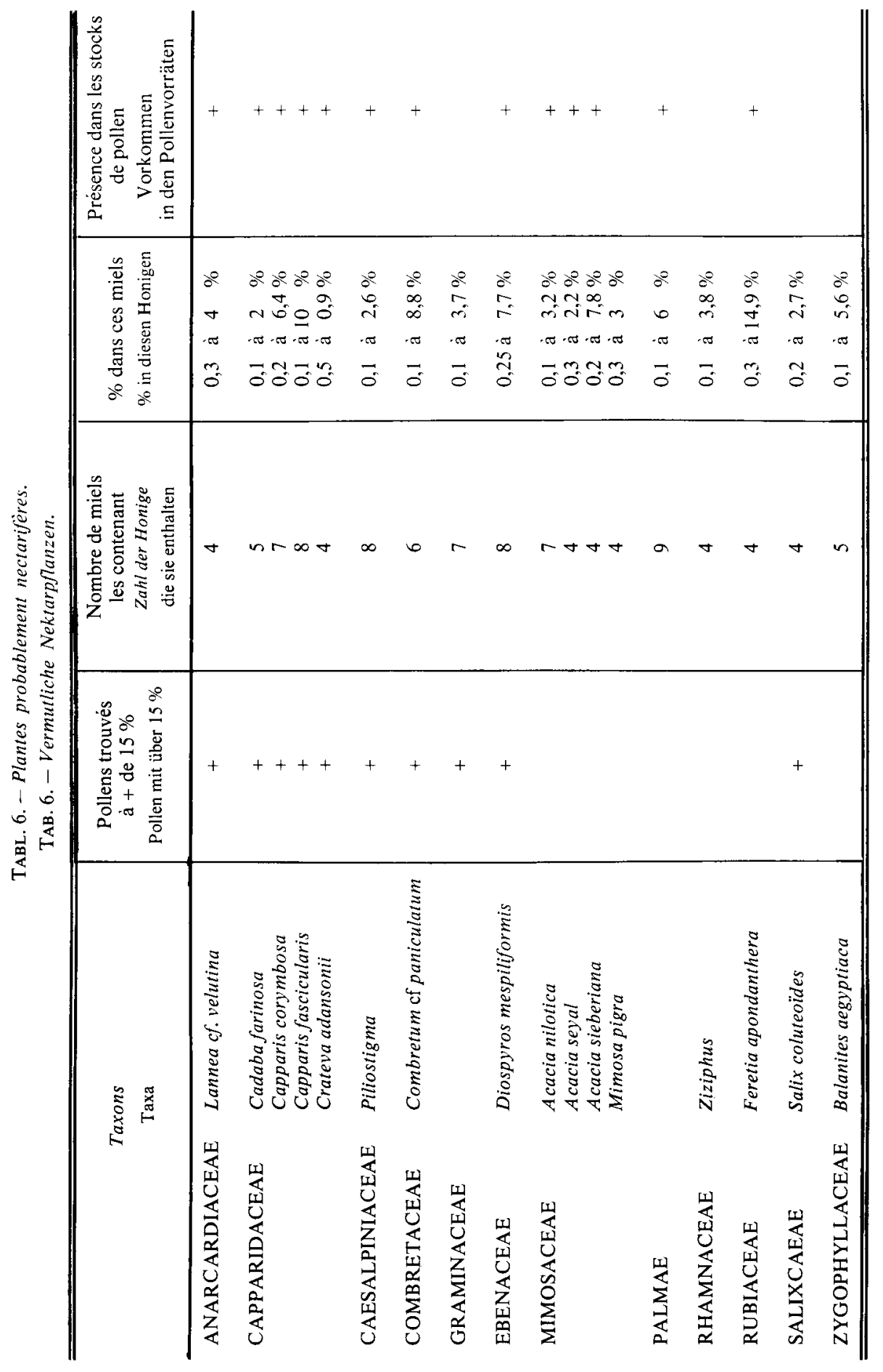




\section{5) Calendrier mellifère}

La confrontation des analyses polliniques effectuées sur les produits récoltés dans les trois villages étudiés met en évidence la difficulté de dresser un calendrier apicole comparable à ceux que l'on peut établir en Europe. En fait, il y a butinage tout au long de l'année pour diverses plantes et notamment Capparis fascicularis. Ceci correspond bien à une caractéristique biologique de beaucoup de végétaux tropicaux qui ont un cycle reproductif peu fixé; certaines espèces montrent, sur un même arbre, simultanément des fleurs et des fruits, tandis que d'autres laissent voir, dans un peuplement monospécifique, des arbres à des stades phénologiques très différents au voisinage les uns des autres (SCHNELL, 1970-1971). Tant que les conditions écologiques le permettent les individus d'une même espèce fleurissent; seule la sécheresse maximale bloque la floraison.

\section{6) Flore mellifère et flore locale}

Ne disposant que d'un nombre d'échantillons relativement restreint, nous ne tenterons pas d'établir des relations entre la composition floristique en chaque site d'étude et les spectres polliniques des miels. Nous nous limiterons à des remarques ponctuelles, toute comparaison globale risquant d'être hâtive ou erronée.

- Au village des Linia, des pollens de type graminéen sont présents dans les miels d'octobre à avril; en outre, un échantillon récolté en décembre renferme $90 \%$ de ce type pollinique. Les autres échantillons en renferment un pourcentage de 0,1 à $9 \%$. Au Kenya Bogdan (1962) a déjà observé ce comportement de butinage de l'abeille domestique sur les graminées.

- A Maïlao, Nymphae est présent dans tous les miels récoltés de janvier à mai. Le nénuphar n'est pas, dans les régions tempérées, une plante mellifère importante. La zone de Maïlao située entre les lits du Logone et du Chari comporte des plaines d'inondations qui voient fleurir sur de grandes surfaces $N y m p h e a$ après la saison des pluies d'octobre à janvier et au début de la suivante en mai. Nymphea se retrouve dans le miel provenant de Linia.

- L'influence humaine sur la végétation et par conséquent sur la flore mellifère s'exprime sous deux formes : les pollens du Goyavier (Psidium gayava) sont assez nombreux $(8,6 \%)$ dans le miel récolté en janvier à Maïlao. Ils signalent la présence de cette espèce au nord de son habitat normal. Or, la goyave est récoltée et consommée par l'homme; le goyavier a été introduit récemment dans cette zone où, bien qu'il soit encore rare, il est butiné sélectivement.।

L'autre effet de l'influence humaine, l'appauvrissement local de la végétation, se traduit dans les différences de structure des spectres polliniques dans les trois villages.

Le nombre moyen de taxons inventoriés diffère d'un village à l'autre : Linia $=8,8$, Rokum $=11,1$, Maïlao $=11$, mais il reste très faible, comparativement au contenu pollinique des miels européens. 
Le nombre total de taxons identifiés varie également : à Linia 26 taxons ont été identifiés, à Maïlao 25 et à Rokum 33. A Rokum, chaque miel pris isolément est plus riche en taxons et cette diversité se retrouve au niveau de l'ensemble de la récolte locale de miel. Or cette zone est située au bord du Chari, dans une région avoisinant la réserve naturelle de la Kalamaloué où la variété des espèces végétales est plus grande que dans les régions voisines. Par contre, les régions de Linia et de Mailao sont beaucoup plus exploitées par l'homme, en particulier pour la fabrication du charbon de bois, qui est très active dans ces deux régions du fait de la demande que représente la capitale, comme le montre l'état de délabrement de la végétation le long des axes routiers conduisant à Linia et à Maïlao.

\section{CONCLUSION}

Le présent travail apporte, dans un domaine de connaissances à peine exploré une somme d'informations scientifiques encore partielle sur les rapports d'Apis mellifica adansonii avec le milieu, au Tchad méridional.

L'étude des composantes polliniques des produits stockés, miels et pollen, permet d'établir un inventaire, dont la brièveté est remarquable, des plantes mellifères du Tchad méridional. Il ne représente quantitativement que $0,03 \%$ de la flore inventoriée actuellement. Parmi ces plantes, on peut désigner les espèces pollinifères, celles-ci étant parfois simultanément nectarifères, et celles qui ne sont probablement que nectarifères.

La structure des spectres polliniques semble en corrélation avec la richesse floristique de chaque lieu. La variation des spectres polliniques au cours de l'année donne une indication sur la phénologie des espèces mellifères, bien que la durée de stockage des pollens dans la ruche soit inconnue. Enfin, certaines espèces dont le pollen est stocké caractérisent la végétation locale, comme les nénuphars des mares d'inondation de Mailao.

La composition pollinique des miels reflète la flore avoisinant le rucher mais montre également le choix très sélectif de l'abeille au sein de cette flore, comme en témoigne, notamment, la récolte du pollen de Goyavier.

Ces données ont été rassemblées dans le cadre d'un projet qui visait à contribuer à l'amélioration de l'apiculture tchadienne, d'une part grâce au développement du rendement des ruchers par intégration des données biologiques et ethnologiques, d'autre part grâce à l'adaptation des méthodes apicoles aux exigences des marchés internationaux et aux possibilités mellifères locales. 


\section{ZUSAMMENFASSUNG}

Diese Arbeit stellt den Beginn eines Inventars der Nektarflora des südlichen Tschad in der Sahel- und Sahelosudan-Zone dar, gelegen zwischen dem 11. und 12. nördlichen Breitengrad (Abb. 1). In dieser Region befindet sich die tropische Flora von (eisenhaltigen) Lateritböden, z. T. semiarid, und besteht vorwiegend aus Steppen von Stachelakazien, gezeichnet von menschlichen Einflüssen, oder aus Waldsavannen mit Überwiegen von Combretaceen.

Dieses Tracht-Inventar beruht auf der gleichzeitigen Anwendung von drei Untersuchungsmethoden : Ethnologische Befragungen über die Erfahrungen der traditionellen Imkerei, direkte Beobachtung der Trachtbienen und schliesslich die Pollenanalyse der Volksreserven (Vorräte an Pollen und Honig). Die Methoden der Analyse und die entsprechende qualitative und quantitative Auswahl sind angemessen.

Die erarbeiteten Resultate sind für das traditionelle Wissen in Tab. I dargestellt, wo 8 Taxa angeführt sind; für die Beobachtungen des Verhaltens die Liste auf Seite 13 mit 25 Taxa; und schliesslich das Ergebnis der Pollenanalyse, welche das vollständigste Verzeichnis ergeben hat : 45 nektarspendende Taxa (Tab. II).

Dieses Teilinventar wird ergänzt durch einen Trachtkalender, welcher die Periode angibt, in der bestimmte Pollen in dem geernteten Honig vorkommen (Tab. IV), sowie eine Liste der Arten, die nur zur Ernte von Pollen besucht wurden (Tab. III).

Die Gesamtheit dieser Resultate zeigt die Bedeutung der von der Biene getroffenen Auswahl aus dem Bereich einer gegebenen Flora : Die Nektarflora des südlichen Tschad, die natürlich ein Bild ciner lokalen tropischen Flora bietet, ist noch viel deutlicher das Ergebnis der Auswahl durch Apis mellifica andansonii aus dieser Flora (der Anteil der Bienenpflanzen beträgt nur $0,03 \%$ der gegenwärtig aus dem Tschad bekannten Pflanzen).

\section{BIBLIOGRAPHIE}

Allan P., 1963. - Pollination of papaws. Fing. S. Afr., 38 (11), 13-15.

Aubreville A., 1949. - Climats, forêts et désertification de l'Afrique tropicale - Paris, Soc. édit. Géogr. marit. et colon., $351 \mathrm{p}$.

Beyleveld G. P., 1968 a. - Nectar and pollen producing trees and plants of the Transvall. S. Afr. Bee J., 40 (4), $10-11$.

Beyleveld G. P., 1968 b. - Detailed list of nectar and pollen producing plants of cconomic importance in the Transvaal. S. Afr. Bee J., 40 (6), 13-14.

Bogdan A. V., 1962. - Grass pollination by bees in Kenya. Proc. Linn. Soc. Lond., 173 (1), $57-60$.

BONNEFILlE R., 1972. - Associations polliniques actuelles et quaternaires en Éthiopie (Vallées de l'Awash et de l'Omo). Thèse Sciences, Paris VI, 1972, 513 p., XIX pl.

Chandler N. T., Mdemu E. M., 1974. - Pollination research in East Africa problems and promises. III rd. Int. Symp? Pollination, 15-18 May 1974, Prague, 57-65.

Chapman G. P., 1964. - Pollination and the yield of tropical crops : an appraisal. Euphytica, 13, 187197.

Crane E., 1973. - Honey sources of some tropical and subtropical countries. Bee Wld., 54 (4), 177-186.

DE VRIEs C. A., 1975. - Bee-keeping in the tropics. Abstr. tropical agriculture, 1 (6), 9-23.

Dubors L., Collart E., 1950. - Beekeeping in the belgian Congo and in Ruanda-Urundi. Bruxelles, Ministère des Colonies, 203 p.

Erdtman G., 1952. - Pollen morphology and plants taxonomy. - Stockholm, Almquist et Wiksell, 539 p. Fotius G., 1973. - Étude phytosociologique du triangle Fort-Lamy, Bousso, Laï (République du Tchad). Fort Lamy, ORSTOM, $226 \mathrm{p}$.

Free J. B., 1974. - Observations on the pollination of Papaya (Carica papaya L.) in Jamaïca. Trop. Agric. Trin., 52, 275-279. 
FreE J. B., 1976. - Insect pollination of tropical crops. Ilford, Central Association of bee-keepers, $10 \mathrm{p}$. GADBIN C., 1976. - Aperçu sur l'apiculture traditionnelle dans le sud du Tchad. J.A.T.B.A., XXIII (4-56), 101-115.

Gadbin C., 1979. - L'intérêt de l'acétolyse en mélissopalynologie. Apidologie, 10, 23-28.

GalIL J., Zeron M., 1967. - On the pollination of Ziziphus Spina-Christi I. Willd. J. Bot., 16, 71-77. GASTON A. et al., 1976. - Pâturages $d u$ sud-ouest du Tchad - Ndjamena, I.E.M.V.M.P.T., 2 vol., 500 p.

GuY R. D., 1972. - The honey hunters of southern Africa. Bee Wld., 53 (4), 159-166.

HAMmons R. O., LeUCK D. B., 1966. - Natural cross pollination of the peanuts, Arachis hypogaea L., in the presence of bees and trips. Agron. J., 58, 396.

Lebrun J.-P., ANDRU J., Gaston A., 1972. - Catalogue des plantes vasculaires du Tchad méridional. Maisons-Alfort, I.E.M.V.P.T., 289 p.

LePISSIER J., 1968. - L'apiculture en République Centrafricaine. Ronéoté, 120 p. R.C.A. : Ministère de l'Équipement.

Letouzey R., 1968. - Étude phytogéographique du Cameroun. - Paris, P. Le Chevalier, $508 \mathrm{p}$.

LiNDER J., 1967. - Étude sur l'apiculture au Sénégal. État d'Israël, Ministère des Affaires étrangères, 14 p.

Louveaux J., Maurizio A. and Vorwohl G., 1978. - Methodes of melissopalynology Bee Wld., 59 (4), 139-157.

McGregor S. E., 1974. - Insect pollination of tropicals crops. III. Symp. intern. Pollinisation, Prague, 1974. Suppl. B.T.A., 2, 1975, 47-55.

Mahadevan V., Chandy K. C., 1959. - Preliminary studies on the increase in cotton yield due to honeybee pollination. Madras. agr. J., 46 (1), 23-26.

Maurizio A., 1968. - La récolte et l'emmagasinage du pollen par les abeilles. Traité de Biologie de l'Abeille, T. III, 168-173.

Maurizio A., Louveaux J., 1965. - Pollens des plantes mellifères d'Europe. Paris, Union des groupements apicoies français, $148 \mathrm{p}$.

N’DiAYe M., 1974. - L'apiculture au Sénégal. Dakar, 134 p. ronéo. Thèse vét. Dakar (1973-74) nº 3.

REILle M., 1970. - Étude pollenanalytique de tourbières du Maroc. Recherches préliminaires et premiers résultats. Thèse spécialité, Marseille, 1970.

Rosario Nuñes J. F., Tordo G. C., 1960. - Prospects and research in beekeeping in Angola, Lisboa. Junta de Investigasões do Ultramaï, $186 \mathrm{p}$.

SCHNELl B., 1970-1971. - Introduction à la phytogéographie des pays tropicaux - 2 vol. Paris, GauthierVillars.

SMITH F. G., 1957. - Bee Botany in East Africa. E. Afr. agric. J., 23 (2), 119-126.

SMITH F. G., 1960. - Beekeeping in the tropics. Bristol, Longmans.

Sowunmi M. A., 1976. - The potential value of honey in paleoecology and archeology. Rev. Paleobotany Palynology, 21 (2), 171-185.

Vergeron P., 1964. - Interprétations statistiques des résultats en matière d'analyse pollinique des miels. Annales Abeille, 7 (4), 349-364. 